\title{
An Outlook on Silicon Nanocrystals for Optoelectronics
}

\author{
I. Capan ${ }^{1}$, A. Carvalho ${ }^{2}$ and J. Coutinho ${ }^{3, *}$ \\ ${ }^{1}$ Rudjer Boskovic Institute, P.O. Box 180, 10000 Zagreb, Croatia \\ ${ }^{2}$ Graphene Research Centre and Department of Physics, National University of \\ Singapore, 117542, Singapore \\ ${ }^{3}$ Department of Physics and I3N, University of Aveiro, Campus Universitário de \\ Santiago, 3810-193 Aveiro, Portugal \\ *Corresponding Author: jose.coutinho@ua.pt
}

Received 5 November 2015; Accepted 3 June 2016;

Publication 1 August 2016

\begin{abstract}
Silicon nanocrystals have emerged as promising material components which extend the realm of the application of its bulk counterpart beyond traditional boundaries. Over the last two decades of research, their potential for application on areas that range from optoelectronics to information storage has been progressively unraveled. Nevertheless, as technology steps forward, new challenges are arising. Here we consider what has been achieved and what are the current limitations on the fields of growth, characterization and modeling of silicon nanocrystals.
\end{abstract}

Keywords: Characterization, modelling, nanocrystals, silicon.

\section{Introduction}

Nanocrystals (NCs) have emerged as a successful route to control and access quantum phenomena. They are able to provide novel NC-based materials with unprecedented and spectacular properties, applicable to a wide field of areas ranging from solar cells, memory devices, thermoelectrics, light emitters, spintronic devices or printable electronics.

Journal of Green Engineering, Vol. 5, 49-70.

doi: 10.13052 jge 1904-4720.5344

(c) 2016 River Publishers. All rights reserved. 
The use of semiconductor NCs instead of two dimensional floating gate devices like in standard complementary metal-oxide-semiconductor (CMOS) field effect transistors and memory devices, makes charge storage less sensitive to common problems such as leakage current and dielectric breakdown. At the same time allows an ultimate miniaturization, and consequently huge savings in power consumption, without electrical instabilities [1]. In particular, nanostructures offer significant breakthroughs in photovoltaics as they can easily overcome the thermodynamic efficiency limit of a conventional junction cell. In fact, it has been anticipated that quantum dot (QD) solar cells could reach power conversion efficiencies of up to 66\% [2]. Much of this improvement comes from the ability of NCs to harvest light with specific wave-lengths, depending on their size, shape, or for example their surface coverage. A colloidal nanocrystal solar cell which combines all the advantages of organics (scalable and controllable synthesis) with transport properties comparable to traditional photovoltaic semiconductors has been demonstrated [3]. Different approaches have also been made to achieve light emission from group-IV semiconductor nanostructures. Despite the indirect nature of the band gap in bulk silicon, quantum confinement of electronic states in sufficiently small NC makes them essentially direct gap structures. This has led to efficient luminescence and electroluminescence of semiconductor nanostructures, including Si- and Ge-NCs embedded in an oxide matrix [4].

Theoretical research on group-IV semiconductor NCs was fundamentally triggered by interest in their optical properties [5-7], particularly after a demonstration of quantum confinement in Si-NCs by Furukawa and Miyasato [8]. These authors reported the opening of the optical gap of small Si dots by more than $100 \%$ with respect to bulk Si. Within a "particle-in-a-box" description, the effect was simply cast as a power law $E_{\text {gap }}=E_{\text {bulk }}+\alpha / R^{n}$ [9], where $R$ is the particle size, $\alpha$ is a confinement factor and $n$ is usually $1<n<2$, depending mostly on the surface termination and host material (for a detailed description see Ref. 10). Despite its popularity, such an empirical approach overlooks important physical aspects such as the chemical nature of the NCs or the dielectric mismatch across their boundaries [11].

One important issue that has led to considerable debate relates to the screening effects within the NCs [12-15]. An accurate theory for the dielectric screening is essential to understand the response of nanostructures and nanostructure ensembles to an external electro-magnetic field. Screening is also at the realm of electrical doping. It is now consensual that even for NCs with a diameter as small as $2 \mathrm{~nm}$, the spatially averaged electronic screening within the core is virtually identical to that in bulk, decreasing 
to the vacuum value close to the polarized surface [16]. Confinement due to underscreening is essentially a surface/interface effect that manifests itself when the surface-to-volume ratio of the structures becomes large enough [14].

Many important questions related to impurities and surface chemistry are yet to be answered. In this respect, quantum-chemical modeling can play a decisive role. Atomistic-scale density functional theory (DFT) calculations of NC materials have been hampered for several reasons. Possibly the most important one stems from the sheer size of the problem. However, with the development of efficient linear-scaling density functional methods along with the steady drop of CPU-time costs, we are now able to solve by first-principles the all-electron problem of systems with a few thousands of atoms [17]. This has led to the understanding of more complex problems such as doping [18-20] and electronic transport [21,22]. These are among the advances that will be addressed below.

\section{Growth and Characterization}

Among all semiconductor nanostructures for photovoltaics, the Si-NCs embedded in a $\mathrm{SiO}_{2}$ matrix have been the most studied in the last few years. One of the first approaches was to form layered structures, so-called superlattices, of Si-NCs produced by reactive evaporation [23]. Reactive evaporation, magnetron co-sputtering and ion implantation are techniques which enable us to control the size, ordering and space distribution of the embedded NCs. These growth techniques are briefly revised below, along with their benefits and limitations. However, it should be noted, that other important and wide-spread techniques are also used for the growth of Si-NCs, namely plasma-enhanced chemical vapor deposition (PECVD) and molecular beam epitaxy (MBE). From the experimental point of view, the synthesis of free-standing NCs will not be covered within this review. However, those issues are covered in the Section 3 of this paper, which deals with the first principles modelling of the NCs.

\subsection{Magnetron Co-sputtering}

Magnetron co-sputtering is a technique for thin film deposition. Together with thermal evaporation and pulsed laser deposition (PLD), they are all fabrication methods based on the production of sub-stoichiometric oxides, with thermal evaporation being the simplest among them. The main difference between these techniques is the exact stoichiometry of the oxides produced. 
In particular, silicon oxide is thermodynamically unstable, and subsequent annealing will yield to the formation of Si-NCs embedded in silicon dioxide.

As already mentioned, the first super-lattice approach for Si-NC formation was achieved by evaporation. Lately, the magnetron co-sputtering has become the most used technique for such structures.

The NC's size, distribution and shape can be easily controlled by varying sputtering time, composition of sputtered material, as well as the annealing temperature, time and atmosphere. Since NCs embedded in the oxide matrix have a large interface area-to-volume ratio, they show a high density of interface defects [24]. In such cases, the role of the interface and interface-related traps cannot be neglected.

The interface traps are located at the substrate/oxide matrix interface (most commonly $\mathrm{Si}_{-} \mathrm{SiO}_{2}$ ) and at the $\mathrm{NC} /$ oxide matrix interface. Unlike the other traps located in the oxide matrix (fixed and mobile oxide charges), interface traps are in electrical communication with the underlying substrate, and therefore largely affect the electrical properties of such structures [25]. Passivation of deep levels caused by the interface is necessary before any NC-based devices become a reality. Vacuum annealing, which is commonly used for NC crystallization, is known to increase the density of the interface traps. However, most of the interface traps can be neutralized by means of a low-temperature $\left(450^{\circ} \mathrm{C}\right)$ anneal in a hydrogen or forming gas [25].

PLD is another technique for the deposition of high quality oxide thin films, and a huge effort has been devoted to the production of Si-NCs by this way. Martin-Sanchez et al. [26] have reported that crystallization of asdeposited amorphous Ge-NCs can be achieved by a relatively low and short temperature treatment, which significantly decreases the thermal budget, when compared to other growth techniques. Moreover, it implies that PLD should be considered as an excellent alternative to widely used magnetron co-sputtering technique for the deposition of complex oxide thin films and NCs. Another variation of this technique, the pulsed laser ablation (PLA) in liquid media has attracted a lot of interest in the recent years. The advantages of the PLA are numerous but still not fully exploited. In particular, PLA in liquid media is a very simple, fast and environment-friendly technique for the synthesis of colloidal semiconductor nanoparticles.

\subsection{Ion Implantation}

The synthesis of semiconductor NCs by means of ion beams has been extensively studied in the past years [24]. NC size, shape and distribution 
can be controlled by varying the implantation conditions (implantation energy and dose) and subsequent annealing. A huge effort has been devoted to the understanding of the energy loss process and defect formation in implanted materials.

The ion implantation introduces defects in materials, and it is a well-known fact that $\mathrm{Si}$-ion implantation of $\mathrm{SiO}_{2}$ is characterized by the production of a large number of oxygen vacancies and other defects in the oxide matrix. These defects play a crucial role in the process as they enhance the $\mathrm{NC}$ formation and the formation of the sub-oxide interface states [24].

Ion implantation is inseparable from CMOS technology, and as such, it is desirable to use it not only for NC fabrication but for the doping of NCs as well. Doping of the NCs is one of the still unresolved issues which limits the applicability of NCs in optoelectronics. Over the past decade, phosphorus and erbium doped Si-NCs have attracted a great deal of attention, due to the promising optoelectronic applications from solar cells to optical amplifiers in telecommunications [27]. It has been shown that in order to study the donor doping and dependences of the photoluminescence (PL) intensity in such systems, it is crucial to understand and control the presence of defects. The effects of donors and defects to the PL of Si-NCs are strongly overlapping [28]. Moreover, it was reported that the decrease in the density of interface related defects may be produced by light phosphorus doping [29].

Another approach for doping semiconductor NCs could be found among techniques already used for doping their bulk counterparts, like neutron transmutation doping (NTD). NTD is a technique commonly used to dope bulk silicon, but for the NCs is not much explored. Recently, the promising application of the NTD for arsenic doping of Ge-NCs has been reported [30].

\subsection{Properties and Characterization}

To obtain all necessary information regarding the properties of NCs and their possible applications, it is desirable to perform a complete characterization that includes structural, optical and electrical specifications. Most characterization reports on semiconductor NCs start with transmission electron microscopy (TEM) data. This is a widespread and well-known technique, which gives extremely valuable structural information. TEM has been used to study $\mathrm{Si}-\mathrm{NCs}$ produced by reactive evaporation in one of the first approaches in producing embedded semiconductor NCs, the so-called superlattice approach [23]. This method enables an easy and well-defined control over the particle size, density and ordering. 


\section{I. Capan et al.}

Along with TEM, there are other useful techniques that can provide information regarding the structural properties of NCs. One of them is grazing incidence small x-ray scattering (GISAXS), which is a non-destructive technique for structural characterization of NCs supported on a substrate [31] and NCs embedded in a matrix [32]. The Si-NCs formed in the super-lattice structures have been successfully studied by means of GISAXS despite the fact that the difference in electronic density (on which GISAXS is very sensitive) between Si-NCs and silicon oxide is very small [33]. Figure 1 shows the schematic representation of a typical experimental GISAXS setup, with details given elsewhere [34]. A two-dimensional CCD detector placed perpendicular to the incoming beam (grazing angle of incidence) is used to record 2D GISAXS pattern. From the measured 2D GISAXS patterns, it is possible to determine the size, shape, inter-NCs distance and size distribution of NCs.

Among all properties of semiconductor NCs, the optical properties have been the most extensively studied, usually by PL. Si-NCs often show strong luminescence intensity in the visible and near-infrared region. A sizedependent blue shift of the luminescence comparable to porous $\mathrm{Si}$ is well

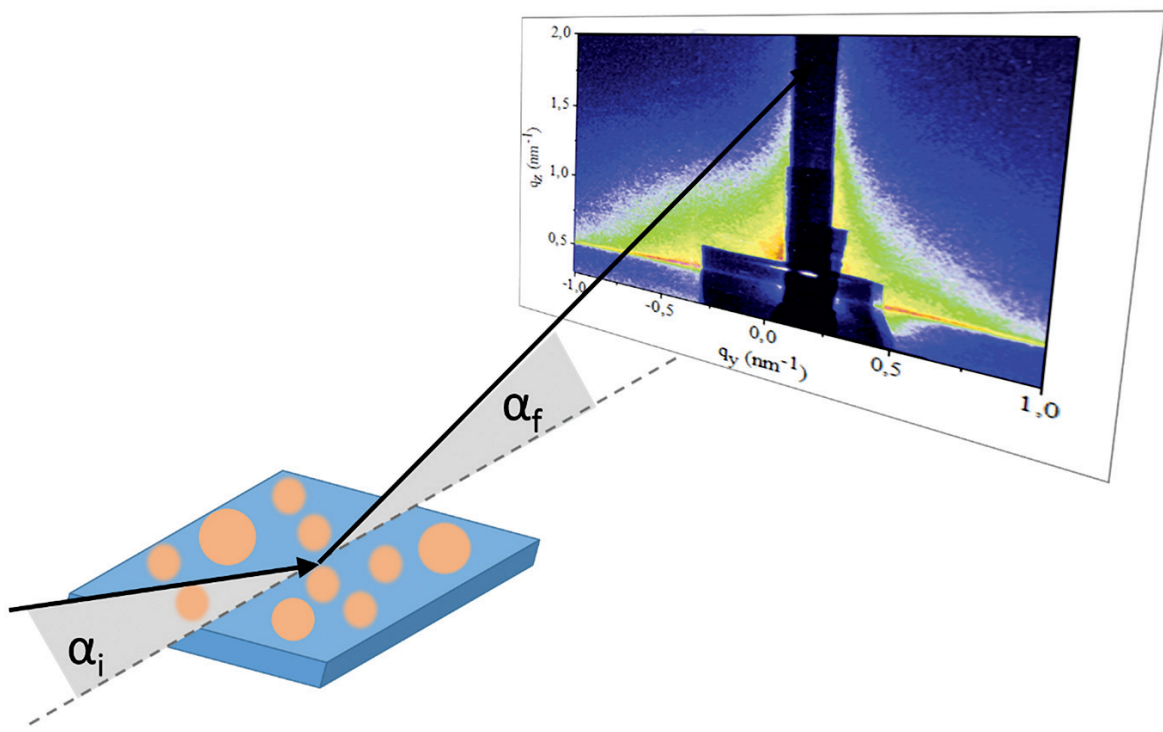

Figure 1 Schematic representation of the GISAXS experiment. The sample is placed horizontally. The incident angle is denoted as $\alpha_{i}$. A 2D CCD detector is positioned perpendicular to the incident beam, and it is used to record SAXS intensity (so called 2D GISAXS pattern). A thin Al-strip (beam stopper) is usually placed in front of the central part of the 2D detector to avoid the overflow of the detector. 
documented. Interaction of Si-NCs with Er ions has attracted a lot of attention due to the possibility of operating in the telecommunications band around $1.5 \mu \mathrm{m}$. Recent studies have shown that phosphorus doping can result in either a quenching or an enhancement of the Si-NC's PL, depending on the dopant concentration and NC sizes [28, 35]. Crowe et al. [35] suggested the existence of competing pathways for the donor electron, which depend strongly on the $\mathrm{NC}$ size. For relatively small NCs, the tendency of phosphorus to accumulate at the nanocrystal-oxide interface results in a passivation of dangling bond type defects as evidenced by an enhancement of the integrated intensity and corresponding blue-shift of the emission peak. As the density of large NCs increases at the expanse of the smaller NCs, the majority of phosphorus atoms occupy substitutional lattice sites within the NCs and the luminescence enhancement rapidly diminishes.

To check the transport properties of the embedded Si-NCs and their charge trapping properties, different electrical characterization techniques could be applied. The most common are current-voltage (I-V) and capacitancevoltage $(\mathrm{C}-\mathrm{V})$ techniques. The I-V measurements have been widely used in order to investigate the mechanism of conductivity of the embedded Si-NCs. The direct tunneling (DT), trap-assisted tunneling (TAT), FowlerNordheim tunneling (FN), Poole-Frenkel (PF) conduction or space charged limited current (SCLC) are mostly applied for describing the charge transport properties [36]. Transport properties are affected by the NCs size and space distribution. Zhou et al. [37] have studied the influence of the Si-NC's diameter on charge transport, and explained the influence by the percolation-hopping conduction mechanism. Figure 2 shows a typical I-V measurement at RT for the embedded Si NCs. NCs are formed by the $\mathrm{Si}^{+}$ion implantation in the $\mathrm{SiO}_{2}$ layer grown on the $\mathrm{Si}$ substrate and the subsequent annealing at $900^{\circ} \mathrm{C}$. Metal-oxide-semiconductor (MOS) structures are prepared by thermal evaporation of gold through the masks.

Moreover, $\mathrm{C}-\mathrm{V}$ gives valuable information as far as interface-defects and oxide defects are concerned. There are numerous studies on the charge trapping properties of embedded NCs. However, it should be noted that in a significant number of those reports, effects of defects (either interface-related or in the oxide) have been completely neglected. As some studies indicate $[1,26]$ these issues should be properly considered.

To obtain even more information about defects in embedded NCs and at their interfaces, deep level transient spectroscopy (DLTS) can be applied. DLTS is a well-established technique which is commonly used in studying trap states in the bulk semiconductors [38], and has also been successfully 


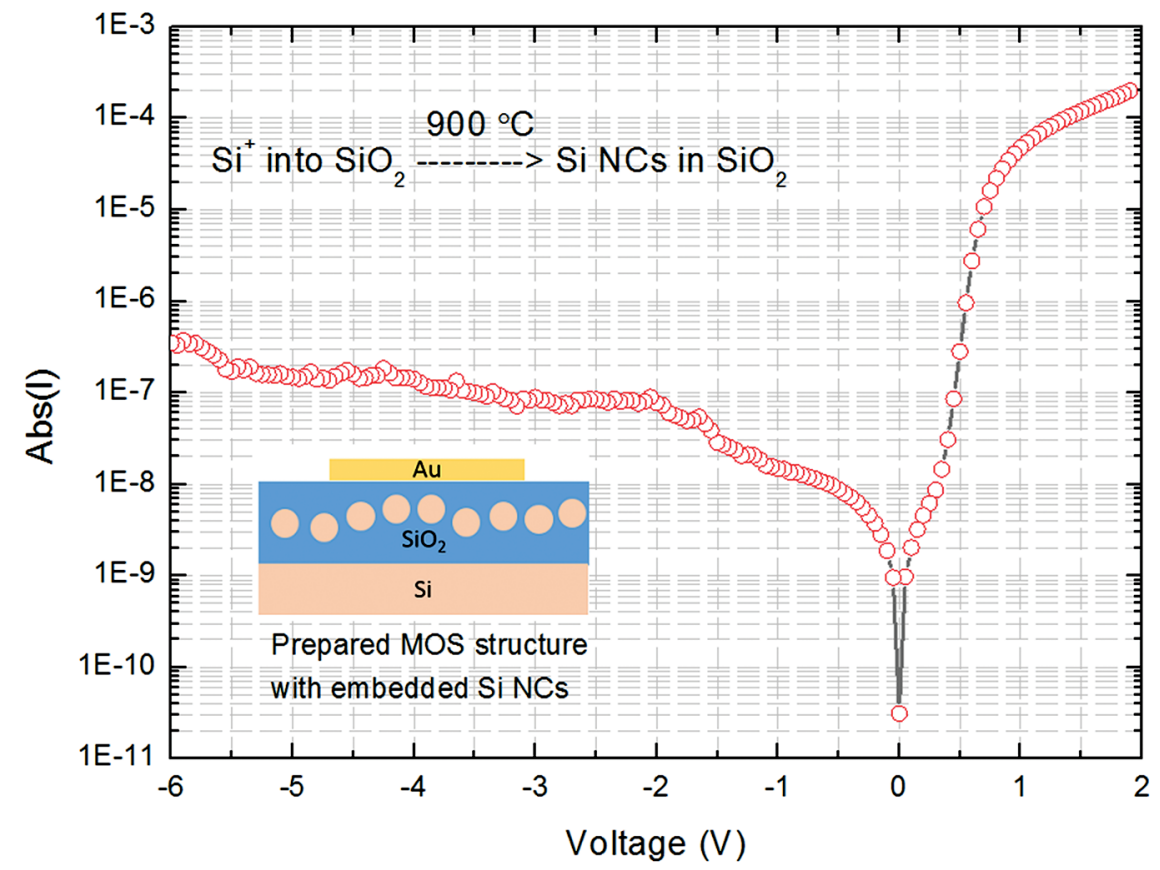

Figure 2 A typical I-V measurement performed on the MOS structure, with embedded $\mathrm{Si}$ $\mathrm{NCs}$ in $\mathrm{SiO}_{2}$, at RT. NCs are produced by ion implantation and subsequent annealing.

applied in studying traps at the $\mathrm{Si}_{-} \mathrm{SiO}_{2}$ interfaces [39] and within NCs [40, 41]. Accordingly, electrons/holes can be thermally emitted out from the NCs and then be detected by DLTS only when their electronic states are lifted above/below the bulk Fermi level.

Another open and still unresolved problem is the electrical transport across doped NC films. Here significant progress has been made in free-standing Si-NC films [22], but much more effort is needed, for instance by using different electrical characterization techniques, to understand the electrical properties of doped and embedded NCs.

\section{Theoretical Modeling of Nanocrystals}

Effective mass models and more elaborate $\boldsymbol{k} \cdot \boldsymbol{p}$-theory were widely employed to understand a several phenomena, including quantum confinement [42], dispersion, as well as strain and electric field dependence of the electronic levels of nanocrystals and quantum dots [43]. Despite being helpful towards grasping 
of trends found by experiments, these techniques lack atomic resolution and overlook many interesting effects.

More recently, significant understanding of the relationships between the atomic structure, chemistry and electronic structure have been obtained from first-principles calculations based on DFT. In analogy to nanostructures grown in a liquid solution or within a solid matrix, nanocrystal models are usually divided into free-standing and embedded. The first class of models mimics the NCs grown, for example, by solidification from plasma or liquid phases, while the second class comprises embedded structures, for example Si- or Ge-NCs in a $\mathrm{SiO}_{2}$ amorphous matrix.

Free-standing nanocrystals in vacuum can be modeled using open boundary conditions i.e. requiring the wavefunction to vanish far from the nanocrystal. An alternative approach is to impose periodic boundary conditions ("particle-in-a-box" approach). This has the advantage of allowing for expansion of the charge density in plane-waves and the use of numerical approaches developed for crystalline solids. However, care has to be taken to ensure that there is little interaction between periodic images of the system. This is particularly relevant when treating systems with a finite electric charge or dipole [44].

Embedded nanocrystals are best modeled using periodic boundary conditions. In that case, one of the main difficulties consists of reproducing the correct structure of the interface and oxidation numbers [45]. The gap between such idealized models, which provide insight into quantum effects, and their respective real-life systems is difficult to bridge. In this respect, there have been some attempts at building multi-scale modeling frameworks [46]. Still, there is ample room for improvement of the description of growth, interaction with solvents, and other multi-scale phenomena.

\subsection{The Many-body Problem}

In the absence of external fields, the Schrödinger equation for a non-relativistic and stationary problem involving a set of $N$ electrons and $M$ atomic nuclei can be written as

$$
\hat{H} \Psi(\mathbf{R})=E \Psi(\mathbf{R}),
$$

with $\mathbf{R}$ representing all electronic and nuclear degrees of freedom (we drop electronic spins for the sake of simplicity). The Hamiltonian in Eq. 1 contains the usual kinetic and potential terms, 


$$
\begin{aligned}
\hat{H}= & -\frac{1}{2} \sum_{i=1}^{N} \nabla_{i}^{2}-\sum_{\alpha=1}^{M} \frac{1}{2 m_{\alpha}} \nabla_{\alpha}^{2}+\frac{1}{2} \sum_{i \neq j}^{N} \frac{1}{\left|\boldsymbol{r}_{i}-\boldsymbol{r}_{j}\right|} \\
& -\sum_{i=1, \alpha=1}^{N, M} \frac{Z_{\alpha}}{\left|\boldsymbol{r}_{i}-\boldsymbol{R}_{\alpha}\right|}+\frac{1}{2} \sum_{\alpha \neq \beta}^{M, M} \frac{Z_{\alpha} Z_{\beta}}{\left|\boldsymbol{R}_{\alpha}-\boldsymbol{R}_{\beta}\right|},
\end{aligned}
$$

with $\boldsymbol{r}_{i}$ and $\boldsymbol{R}_{i}$ representing electronic and nuclear coordinates, respectively, whereas $m_{\alpha}$ and $Z_{\alpha}$ stand for the mass and atomic number of each nucleus, respectively. Disregarding any analytical solution for the above equation, (only known for problems such as the hydrogen and $\mathrm{He}^{+}$atoms [47]), the fact that $\Psi$ depends on at least $3 M+3 \sum_{\alpha} Z_{\alpha}$ Cartesian coordinates makes the simplest of the problems intractable, even when the fastest supercomputers are put at our disposal.

The greatest challenge associated with modeling NCs is the great number of atoms required for the model to meet the sizes of even the smallest particles realized experimentally (about 1000 atoms for a $3 \mathrm{~nm}$ diameter NC). Among the several terms in the many-body Hamiltonian, the electronelectron interaction is the hardest do obtain as it scales with $N^{4}$. This is where DFT comes into play. As opposed to the Schrödinger approach, the Hamiltonian is replaced by a total energy functional $E[n]$ and $\Psi$ by the electron density $n(\boldsymbol{r})$,

$$
E[n]=F[n]+\int \mathrm{d}^{3} \boldsymbol{r} v_{\text {ext }}(\boldsymbol{r}) n(\boldsymbol{r}),
$$

with $v_{\text {ext }}$ representing an external potential to which the electrons are subjected (see for example Ref. 48 for a review on this method). This includes electron-ion interactions and others, such as applied electrical and magnetic fields. The functional $F[n]$ is universal (system-independent), and accounts for the electronic kinetic energy, as well as electronic exchange-correlation interactions [49].

The striking feature of DFT, is that despite the huge simplification of adopting the electron density as the variational variable, no approximations are made. The drawback is that DFT is formally valid for the ground state only, and the exact form of $F[n]$ is unknown. Despite that, several approximations for $F[n]$ have been proposed, with increasing degree of complexity as they improve in the description of the non-local properties of the electron-electron interactions.

Various DFT implementations using localized basis sets e.g. AIMPRO [17, 50], Conquest [51], Onetep [52], OpenMX [53] and SIESTA [54] exhibit order- $N$ scaling [55] and are advertised as enabling calculations with up to 
$N \sim 10^{4}$ atoms. Larger systems have been treated using tight binding or semiempirical methods [56].

Further, atomic-like basis sets, such as Gaussian-type orbitals, are convenient in the case of free-standing nanocrystals, both because they can be naturally combined with open boundary conditions and simultaneously they lead to sparse Hamiltonian and overlap matrices that can be efficiently diagonalized. Notwithstanding, many implementations of density functional theory traditionally used in solid state physics rely on the use of planewave basis sets for expansion of wavefunctions and charge density. Although more robust than local-basis implementations, plane-wave codes have the disadvantage that to make the basis size finite it is then necessary to impose periodic boundary conditions, allowing for sufficiently large vacuum spaces in between a nanoparticle and its image. The large supercell size reflects then into a great number of plane waves, which is independent on the ratio between the volumes of matter and vacuum.

\subsection{Calculation of Optical Properties}

The indirect band-gap nature of bulk Si has been one of the main handicaps of this material. It has placed $\mathrm{Si}$ away from many optical applications like lasers and LEDs, in favor of the more expensive and less environmental friendly III-V semiconductors. While radiative recombination on compound semiconductors is a relatively efficient process, in $\mathrm{Si}$ such transitions are rather unlikely to take place as the large $\boldsymbol{k}$-space mismatch between conduction band minimum and valence band maximum states implies the coupling with phonons. On the other hand, the dispersionless and tunable nature of the electronic structure of SiNCs holds many promises in all-Si optoelectronics. In this respect theoretical modeling has provided many insights. Besides the size and shape effect to the energy and rate of optical transitions, the chemical nature of the surface, shell or host where the NC is embedded play a critical role [57]. For instance, Guerra and Ossicini [58] showed that while radiative recombination of $\mathrm{H}$-saturated $\mathrm{Si}$ $\mathrm{NC}$ is strongly dependent on the size of the crystallites, in hydroxyl-terminated or $\mathrm{SiO}_{2}$-embedded NCs the optical yield is mostly conditioned by the fraction of oxygen termination.

First-principles DFT modeling has also been insightful on the study of local polarizations that take place at $\mathrm{Si}-\mathrm{NC} / \mathrm{SiO}_{2}$ interfaces [59]. The abrupt change of chemical species (with different electronegativities) at the edge of the NCs induces charge displacements that can be accounted for by using pseudopotentials. It has been found that polarizations due to the oxide interface 
have two important effects, namely (i) to quench the low energy absorption region and (ii) a blue-shift of some particularly intense transitions.

Another important aspect is the role of defects. Si dangling bonds or radicals are strongly localized being effective traps for both electrons and holes [21]. They are therefore likely to degrade the optical yield. On the other hand, shallower states like those produced at the $\mathrm{Si} / \mathrm{SiO}_{2}$ core-shell interface of oxidized Si-NCs are most likely to shift the absorption/emission spectra with respect to pristine NCs [60].

Inclusion of many-body effects is important especially for small nanoparticles where the exciton binding energy can be large [61]. These can be calculated using the Bethe-Salpeter equation [62]. However, for nanocrystals with radius larger than about $0.6 \mathrm{~nm}$, the self-energy and Coulomb corrections almost exactly cancel, and one-particle calculations actually give accurate values for the excitonic gap [62].

\subsection{Modeling Dopants in Nanocrystals}

Deliberate introduction of alien species into materials lies at the heart of microelectronics. A prototypical example is the replacement of a few lattice sites in a billion in crystalline $\mathrm{Si}$, for instance by phosphorous or boron, to confer good electrical conductivity to an otherwise poor insulator. In the same way, doping NCs will play an analogous role in future artificial solids or meta-materials made of wave-function engineered nanoparticles.

Although many promising applications of Si-NCs rely on the possibility to tune their electronic states by exploring size and surface effects, the fact is that this is only possible in ultra-small NCs where effective electrical doping (which should lead to considerable fraction of ionized dopants at roomtemperature) is yet to be demonstrated. Notwithstanding, the introduction of dopants in Si-NCs has been unequivocally demonstrated, for instance by monitoring the ${ }^{31} \mathrm{P}$ hyperfine splitting of the phosphorous donor state during electron paramagnetic resonance experiments [63], or from photoluminescent transitions of bound excitons to boron [64].

In small NCs, where surface conditions affect the electronic states, theory predicts that phosphorous and boron levels are rather deep with carrier binding energies of the order of $1 \mathrm{eV}$, anticipating serious difficulties in finding suitable dopants to operate at room temperature [21, 65-67].

In this respect other routes for doping have been under investigation, where transfer doping by means of molecules with high electron affinity or low ionization potential stands as a rather promising alternative. For instance, 
(a)

(b)

(c)

(d)

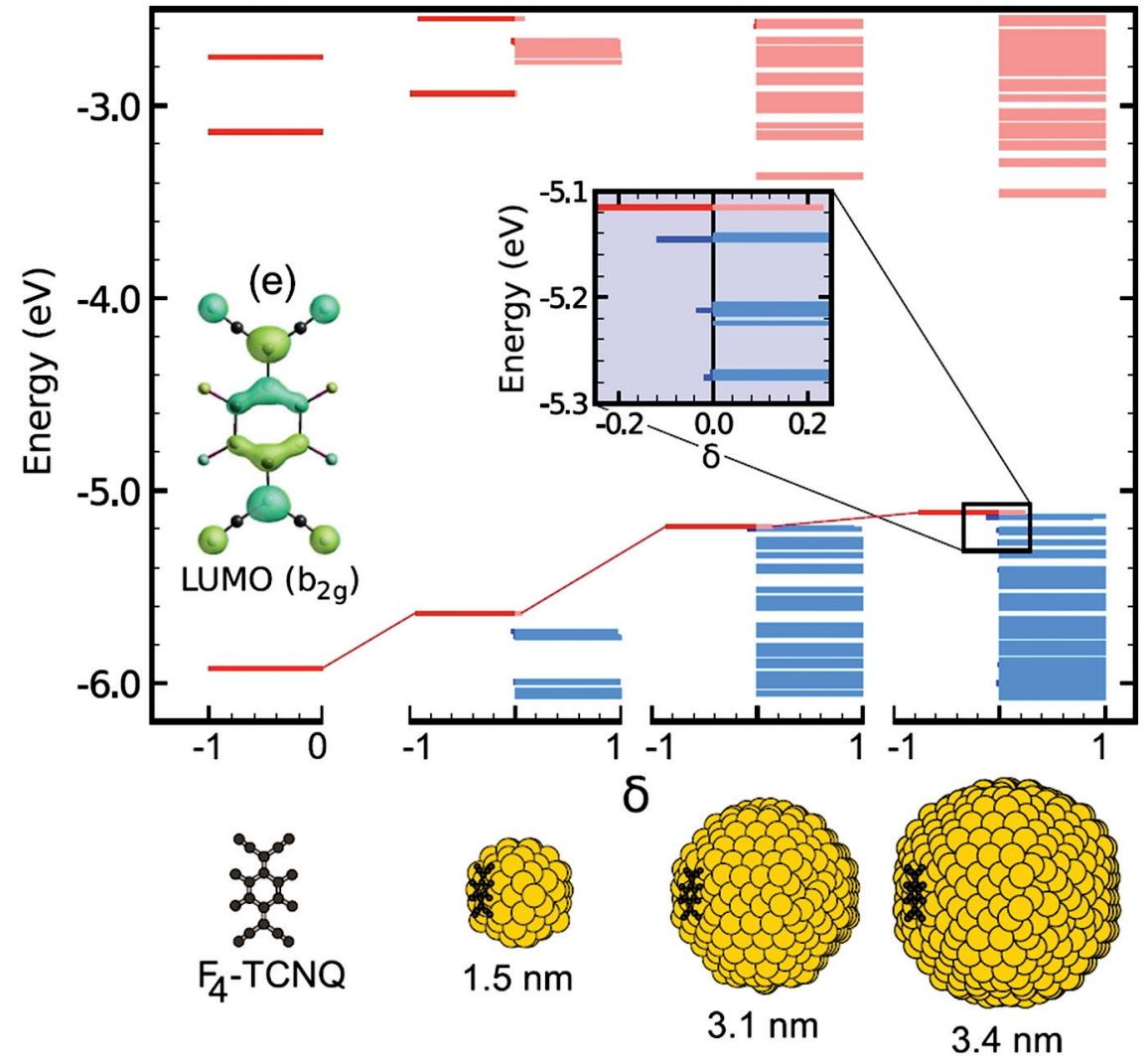

Figure 3 Hybridization between $\mathrm{F}_{4}$-TCNQ and Si-NC one-electron states. (a)-(d) KohnSham eigenvalue energy diagrams and (e) Isosurface plot of the $\mathrm{F}_{4}$-TCNQ lowest unoccupied state ( $b_{2 g}$ symmetry). The energy-level diagrams are for (a) isolated F4-TCNQ and (b)-(d) for F4-TCNQ adsorbed on Si NCs of increasing size. Each state is represented by a bar of unitary length, with the abscissa of the left- and right-hand ends of the bar indicating, respectively, the relative localization on the molecule (negative values) and on the NC (positive values). Blue and red bars stand for occupied and unoccupied states, respectively. (Reprinted with permission from Carvalho et al. [20], (c) 2011 the American Physical Society).

In Ref. 20 it is suggested that the $\mathrm{F}_{4}$-TCNQ (7,7,8,8-tetracyano-2,3,5,6tetrafluoroquinodimethane) molecule, which is commonly used as a p-dopant in organic electronics, could be a candidate to produce holes in the Si-NCs, as shown in Figure 4. They also showed that about 3-4 molecules adsorbed to the NC surface are able to produce a positively charged NC, although the 

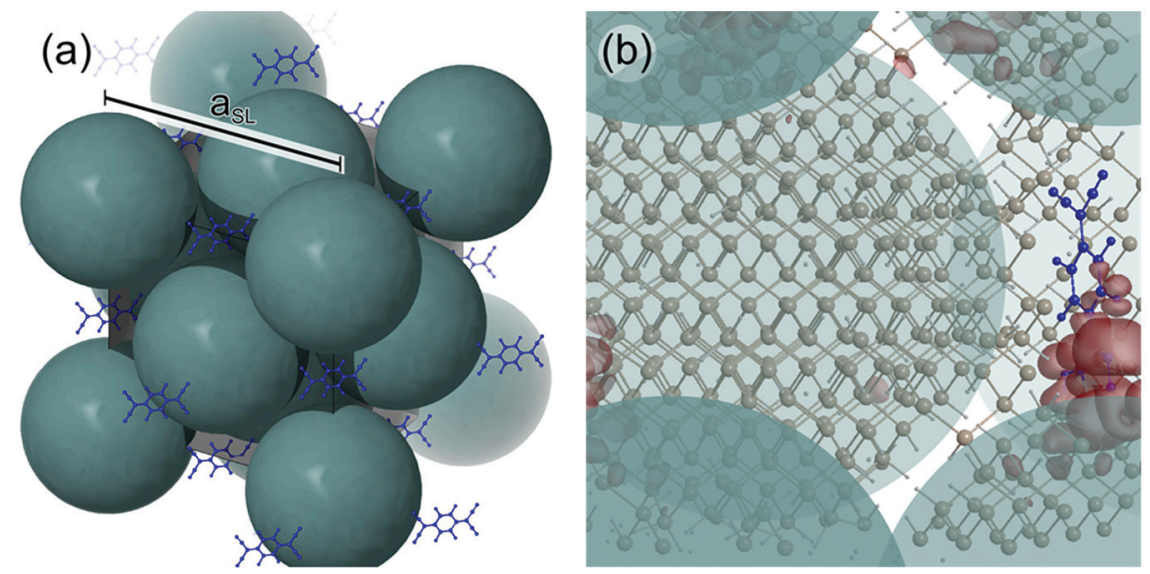

Figure 4 (a) Three-dimensional view of an fcc-superlattice of Si-NCs (large solid spheres) doped with $\mathrm{F}_{4}$-TCNQ molecules (blue). (b) Electron density isosurface (red) of the HOMO level overlapping both the $\mathrm{F}_{4}$-TCNQ molecule and an adjacent Si-NC. Circles enclosing individual NCs are drawn for eye guidance purposes. (Reprinted with permission from Ref. [22]. (C) 2014 American Chemical Society).

first excitation of the doped system was almost $1 \mathrm{eV}$, again indicating that free-holes are unlikely to be produced at room temperature. These results were confirmed recently by means of electrical measurements combined with first-principles calculations [22], demonstrating that $\mathrm{F}_{4}$-TCNQ within a Si-NC solid film is not a shallow acceptor, but rather a deep acceptor with levels that can edge the conduction band minimum of the Si-NC film. In fact, this dopant was used to increase the conductivity of the films by more than 2 orders of magnitude, although the majority carriers were electrons.

\subsection{Surface Properties}

The NC surface is one of the most important variables in the engineering of its shape, intrinsic properties and stability in air and interaction with solvents and solid matrices.

For nanocrystals with a clean surface or with a hydrogen-covered surface, the orientation dependence of the surface energy, as well as edge and corner energies (for less than about 100 atoms) determine the shape of the NCs grown under near-equilibrium quasi-static conditions. It has been found that diamond $\mathrm{NCs}$ are most stable in a truncated octahedral structure, but Si- and Ge-NCs are stable in a nearly-spherical geometry [68]. In any case, free standing $\mathrm{Si}$ and $\mathrm{Ge}-\mathrm{NCs}$ are most often grown in conditions very far from equilibrium, 
justifying the use of spherical models [69]. Diamond nanocrystals however have been grown in different shapes and configurations, and their optical properties have been found to be in discrepancy with theoretical models, presumably due to the contribution of defects [70].

As nanocrystals have a very large surface-to-volume ratio, their electronic and optical properties are largely determined by the surface or interface morphology. Amongst group-IV NCs, Si-NCs are especially reactive in air, immediately forming an oxide cap. Surface oxidation also decreases the gap energy of the NC, red-shifting its absorption and photoluminescence edges [71, 72]. Models of $\mathrm{Si}-\mathrm{NCs}$ embedded in $\mathrm{SiO}_{2}$ also show optical red-shifting following oxidation, and suggest that this effect is associated not only with the decreased quantum confinement, but also with the presence of silanonelike $\mathrm{Si}=\mathrm{O}$ bonds [73]. As a consequence of the change in the electronic structure and dielectric screening, the ionization energy of dopants is also altered. The earliest oxidation stage corresponding to the formation of silanol surface groups was found to increase the electron binding energy of the P, As and $\mathrm{Sb}$, and decrease the hole binding energy of $\mathrm{B}, \mathrm{Al}, \mathrm{Ga}$ and In [74].

Chlorine covered Si-NCs were found to have higher electron affinity, higher ionization energy and lower optical absorption energy threshold than hydrogen covered nanocrystals with the same size [75]. Like the hydrogenated $\mathrm{Si}-\mathrm{NCs}$, chlorinated $\mathrm{Si}-\mathrm{NCs}$ doped with phosphorus or boron require a high activation energy to transfer an electron or hole, respectively, to undoped SiNCs. The electronic levels of surface dangling bonds are similar for both types of surface passivation, although in the chlorinated Si-NCs some fall off the narrower gap. Functionalization with chlorine, nitrogen and fluorine was also found to be an effective way to control the bandgap of nanodiamonds [76].

Nanocrystals functionalized with organic groups have so far only been considered in a limited number of theoretical studies, for silicon [55, 77-79] and diamond [80]. Functionalization with alkyl and aryl molecules is an important preparatory step for the conjugation of the surface to larger organic molecules. Regarding the direct influence on the physical properties, alkyl passivation was found to change little the optical gaps of silicon QDs, but to decrease substantially their ionization potentials and electron affinities and affects their excited state properties. Nevertheless, there is still ample space for the modeling of the interaction of functionalized nanocrystals with solvents, polymers and biological molecules. A tight-binding parameterization has been proposed for this family of systems, offering a cheaper approach that may become a basis for a function-oriented treatment of organic-inorganic interfaces [54]. 


\section{Conclusions}

Although group-IV NCs may emerge as a natural way to extend, enhance and control the properties of their respective bulk materials, the last two decades of research have shown that not only the device architecture, but also the growth, characterization and modeling techniques have to be redesigned to meet their very specific requirements.

Silicon and germanium NCs have revealed to be promising on the areas of optical luminescence and absorption, where size-dependent optical transitions have been consistently reported. This property is particularly appealing from photovoltaics and light emitting devices. Memory devices are another promising area of application. So far, the greatest challenges continue to be the control of surface, interface and other deep levels, misfit strain, and doping efficiency.

From a theoretical point of view, even though one of the most important attributes of nanoparticles - their size-dependent bandgap - is a quantity hard to predict, modeling studies have been able to provide great insight into the fundamental optical and electronic properties of different types of NCs. An open issue still resides on the difficult task of bridging the length scales from the largest sizes achievable numerically to the smallest sizes produced experimentally.

\section{Acknowledgments}

The authors would like to acknowledge the contribution of the COST Action MP1406 MultiscaleSolar. JC thanks support by the FCT through Projects No. PTDC/CTM- ENE/1973/2012 and UID/CTM/50025/2013. IC thanks support by the Croatian Centre of Excellence for Advanced Materials and Sensing Devices.

\section{References}

[1] El Hdiy A., Gacem, K., Troyon, M., Ronda, A., Bassani, F., and Berbezier, I. (2008). J. Appl. Phys. 104, 063716.

[2] Nozik, A. J. (2002). Physica E. 14, 115.

[3] Gur, I., Fromer, N. A., Geier, M. L., and Alivisatos, A. P. (2005). Science $310,462$.

[4] Ray, S. K., Maikap, S., Banarjee, W., and Das, S. (2013). J. Phys. D: Appl. Phys. 46, 153001. 
[5] Wang, L., and Zunger, A. (1994). J. Phys. Chem. 98, 2158.

[6] Öğüt S., Chelikowsky, J. R., and Louie, S. G. (1997). Phys. Rev. Lett. 79, 1770.

[7] Delerue, C., Lannoo, M., Allan, G. (2000). Phys. Rev. Lett. 84, 2457.

[8] Furukawa, S., and Miyasato, T. (1988). Phys. Rev. B 38, 5726.

[9] Timmerman, D., and Gergorkiewicz, T. (2012). Nano. Res. Lett. 7, 389.

[10] Belyakov, V., Burdov, V. A., Lockwood, R., and Meldrum, A. (2008). Adv. Opt. Technol. 2008, 279502.

[11] Franceschetti, A., and Zunger, A. (2000). Phys. Rev. B 62, 2614.

[12] Wang, L., and Zunger, A. (1994). Phys. Rev. Lett. 73, 1039.

[13] Giustino, F., and Pasquarello, A. (2005). Phys. Rev. B 71, 144104.

[14] Delerue, C., Lannoo, M., and Allan, G. (2003). Phys. Rev. B 68, 115411.

[15] Öğüt S., Burdick, R., Saad, Y., and Chelikowsky, J. R. (2003). Phys. Rev. Lett. 90, 127401.

[16] Trani, F., Ninno, D., Cantele, G., Iadonisi, G., Hameeuw, K., Degoli, E., et al. (2006). Phys. Rev. B 73, 245430.

[17] Rayson, M. J., and Briddon, P. R. (2009). Phys. Rev. B 80, 205104.

[18] Ramos, L. E., Degoli, E., Cantele, G., Ossicini, S., Ninno, D., Furthmüller, J., et al. (2008). Phys. Rev. B 78, 253310.

[19] Chan, T. L., Tiago, M. L., Kaxiras, E., and Chelikowsky, J. L. (2008). Nano Letters 8, 596.

[20] Carvalho, A., Coutinho, J., Barroso, M., Silva, E. L., Öberg S., Rayson, M. J., et al. (2011). Phys. Rev. B 84, 125437.

[21] Carvalho, A., Öberg S., Rayson, M. J., and Briddon, P. R. (2012). Phys. Rev. B 86, 045308.

[22] Pereira, R. N., Coutinho, J., Niesar, S., Oliveira, T. A., Aigner, W., Wiggers, H., et al. (2014). Nano Letters 14, 3817.

[23] Zacharias, M., Heitmann, J., Scholz, R., Kahler, U., Schmidt, M., and Blasing, J. (2002). Appl. Phys. Lett. 80, 661.

[24] Barbagiovanni, E. G., Lockwood, D. J., Simpson, P. J., and Gonncharova, V. (2014). Appl. Phys. Rev. 1, 011302.

[25] Schroder, D. K. (2006). 'Semiconductor Material and Device Characterization', (Wiley, New Jersey)

[26] Martin-Sanchez, J., Capan, I., Chahboun, A., Pinto, S. R. C., Vieira, E. M. F., Rolo, A. G., et al. (2013). Appl. Surf. Sci. 280, 632.

[27] Shah, M., Wojdak, M., Kenyon, A. J., Halsall, M. P., Li, H., and Crowe, I. F. (2012). J. Lumin. 132, 3103.

[28] Murakami, K., Shirakawa, R., Tsujimura, M., Uchida, N., Fukata, N., and Hishita, S. (2009). J. Appl. Phys. 105, 054307. 
[29] Mimura, A., Fujii, M., Hayashi, S., Kovalev, D., and Koch, F. (2000). Phys. Rev. B 62, 12625.

[30] Chen, Q., Lu, T., Xu, M., Meng, C., Hu, Y., Sun, K., et al. (2011). Appl. Phys. Lett. 98, 073103.

[31] Kovacevic, I., Pivac, B., Dubcek, P., Zorc, H., Radic, N., Bernstorff, S., et al. (2007). Appl. Surf. Sci. 253, 3034.

[32] Bernstorff, S., Dubcek, P., Kovacevic, I., Radic, N., and Pivac, B. (2007). Thin Solid Films 515, 5637.

[33] Pivac, B., Dubcek, P., Capan, I., Zulim, I., Betti, T., Zorc, H., et al. (2009). Nanosci. Nanotech. 9, 3853.

[34] Kovacevic, I., Dubcek, P., Duguay, S., Zorc, H., Radic, N., Pivac, B., et al. (2007). Physica E 38, 50.

[35] Crowe, I., Papachristodoulou, N., Halsall, M. P., Hylton, N. P., Hulko, O., Knights, A. P., et al. (2013). Appl. Phys. 113, 024304.

[36] Jambois, O., Berencen, Y., Hijazi, K., Wojdak, M., Kenyon, A. J., Gourbilleau, F. et al. (2009). J. Appl. Phys. 106, 063526.

[37] Zhou, X., Usami, K., Rafiq, M. A., Tsuchiya, Y., Mizuta, H., and Oda, S. (2008). J. Appl. Phys. 104, 024518.

[38] Dobaczewski, L., Peaker, A. R., and Bonde Nielsen, K. (2004). J. Appl. Phys. 96, 4689.

[39] Capan, I., Janicki, V., Jacimovic, R., and Pivac, B. (2012). Nuclear Instrum. Meth. Phys. Res. B 282, 59.

[40] Antonova, I. V., Popov, V. I., Smagulova, S. A., Jedrzejewski, J., and Balberg, I. (2013). J. Appl. Phys. 113, 084308.

[41] Buljan, M., Grenzer, J., Holy, V., Radic, N., T. Misic-Radic, Levichev, S., et al. (2010). Appl. Phys. Lett. 97, 163117.

[42] Bimberg, D., Grundmann, M., and Ledentsov, N. N. (1999). Quantum Dot Hetero-structures. Chichester: John Wiley and Sons.

[43] Chang, K., and Xia, J.-B. (1998). J. Appl. Phys. 84, 1454.

[44] Makov, G., and Payne, M. C. (1995). Phys. Rev. B 51, 4014.

[45] Pasquarello, A., Hybertsen, M. S., and Car, R. (1998). Nature 396, 58.

[46] Zahi, I., Mur, P., Blaise, P. H., Estève, A., Djafari Rouhani, M., Vergnes, H., et al. (2011). Thin Solid Films 519, 7650.

[47] J-REF1. Schiff, L. I. (1955). Quantum Mechanics. Singapore: McGrawHill.

[48] Jones, R. O., and Gunnarsson, O. (1989). Rev. Mod. Phys. 61, 689.

[49] Hohenberg, P., and Kohn, W. (1964). Phys. Rev. 136, B864.

[50] Rayson, M. J. (2010). Comput. Phys. Commun. 181, 1051. 
[51] Bowler, D. R., Miyazaki, T., and Gillan, M. J. (2002). J. Phys. Condens. Matter. 14, 2781.

[52] Skylaris, S.-K., Haynes, P. D., Mostofi, A., and Payne, M. C. (2005). J. Chem. Phys. 122, 084119.

[53] OpenMX code. Available at: http://www.openmx-square.org

[54] Soler, J. M., Artacho, E., Gale, J. D., Garcia, A., Junquera, J., Ordejon, P., et al. (2002). J. Phys. Condens. Matter. 14, 2745.

[55] Kohn, W. (1996). Phys. Rev. Lett. 76, 3168.

[56] Trani, F., and Baroni, V. J. (2011). Chem. Theor. Comput. 7, 713.

[57] Puzder, A., Williamson, A. J., Grossman, J. C., and Galli, G. (2002). J. Chem. Phys. 117, 6721.

[58] Guerra, R., and Ossicini, S. (2010). Phys. Rev. B 81, 245307.

[59] Guerra, R., Marsili, M., and Ossicini, S. (2011). Phys. Rev. B 84, 075342.

[60] Koponen, L., Tunturivuori, L. O., Puska, M. J., and Nieminen, R. M. (2009). Phys. Rev. B 79, 235332.

[61] Rajadell, F., Climente, J. I., Planelles, J., and Bertoni, A. (2009). J. Phys. Chem. C 113, 11268.

[62] Delerue, C., Lannoo, M., and Allan, G. (2000). Phys. Rev. Lett. 84, 2457.

[63] Stegner, A. R., Pereira, R. N., Klein, K., Lechner, R., Dietmueller, R., Brandt, M. S., et al. (2008). Phys. Rev. Lett. 100, 026803.

[64] Fujii, M., Hayashi, S., and Yamamoto, K. (1998). J. Appl. Phys. 83, 7953.

[65] Melnikov, D. V., and Chelikowsky, J. R. (2004). Phys. Rev. Lett. 92, 046802.

[66] Cantele, G., Degoli, E., Luppi, E., Magri, R., Ninno, D., Iadonisi, G., et al. (2005). Phys. Rev. B 78, 113303.

[67] Ni, Z., Pi, X., and Yang, D. (2014). Phys. Rev. B 89, 035312.

[68] Barnard, A. S., and Zapol, P. (2004). J. Chem. Phys. 121, 4276.

[69] Mangolini, L., and Kortshagen, U. (2010). Silicon Nanocrystals; Fundamentals, Synthesis, and Applications, eds L. Pavesi and R. Turan (Weinheim: Wiley-VCH).

[70] Landt, L., Klünder, K., Dahl, J. E., Carlson, R. M. K., Möller, T., and Bostedt, C. (2009). Phys. Rev. Lett. 103, 047402.

[71] Vasiliev, I., Chelikowsky, J. R., and Martin, R. M. (2002). Phys. Rev. B $65,121302(\mathrm{R})$.

[72] Zhou, Z., Friesner, R. A., and Brus, L. (2003). J. Am. Chem. Soc. 125, 15599.

[73] Luppi, M., and Ossicini, S. (2005). Phys. Rev. B 71, 035340.

[74] Carvalho, A., Rayson, M. J., and Briddon, P. R. (2012). J. Phys. Chem. C 116, 8243. 
[75] Carvalho, A., Öberg, S., Rayson, M. J., and Briddon, P. R. (2012). Phys. Rev. B 86, 045308.

[76] Brown, N., and Hod, O. (2014). J. Phys. Chem. C 118, 5530.

[77] Reboredo, F. A., Schwegler, E., and Galli, G. (2003). J. Am. Chem. Soc. $125,15243$.

[78] Reboredo, F. A., and Galli, G. (2005). J. Phys. Chem. B 109, 1072.

[79] Zhou, Z., Brus, L., and Friesner, R. (2003). Nano Lett. 3, 163.

[80] Zhong, Y. L., Loh, K. P., Midya, A., Chen, Z.-K., and Suzuki, M. (2008). Chem. Mater. 20, 3137.

\section{Biographies}

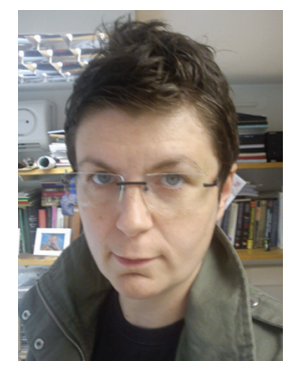

I. Capan was born on 16th of Feb. 1977, Tuzla, Bosnia and Hercegovina. She has obtained her Ph.D. in physics at University of Zagreb, Croatia in 2006. She works at Rudjer Boskovic Institute, Croatia, and currently holds a position of a senior research associate. She was awarded by Chancellor's Award for the Best Student Scientific Work at University of Zagreb in 1999 and National Science Award for Junior Researchers in 2006. Her research is mainly focused on the optoelectronic characterization of semiconductors (from the bulk to the low dimensional structures) and hybrid materials with particular interest in the developing the new generation of devices, from non-volatile memories to solar cells. She has published 50 research papers. 


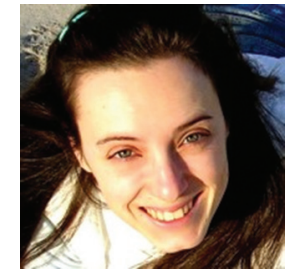

A. Carvalho received her first degree at the University of Aveiro, Portugal, in 2005. This was followed by a Ph.D. at the University of Exeter, UK (2005-2008), where she investigated defects in semiconductors, and an incursion at the physics of ferroelectrics as a post-doctoral researcher at the EPFL, Switzerland. She was awarded a Marie-Curie fellowship at the University of Aveiro, from 2011 to 2012. At present, Alexandra is a Senior Research Fellow at the National University of Singapore.

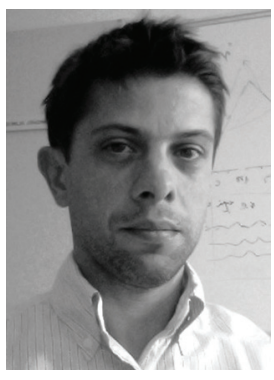

J. Coutinho is a Principal Researcher at the University of Aveiro in Portugal. He did a Ph.D. in Physics at the University of Exeter (UK), and since then he has been involved in several funded projects, many of them in collaboration with experimentalists. His research is mostly theoretical, and involves looking at the fundamental properties of defects in solids, surfaces and nanostructures of semiconducting and insulating materials by means of electronic structure calculations (density functional theoretical methods) and first principles molecular dynamics. He has (co-)authored over 100 papers in peer-review journals (ISI). 
